\title{
RESULTS OF 150 CONSECUTIVE BILATERAL LUNG VOLUME REDUCTION PROCEDURES IN PATIENTS WITH SEVERE EMPHYSEMA
}

Joel D. Cooper, $\mathrm{MD}^{\mathrm{a}}$

G. Alexander Patterson, $\mathrm{MD}^{\mathrm{a}}$

R. Sudhir Sundaresan, MD $^{\mathrm{a}}$

Elbert P. Trulock, $\mathrm{MD}^{\mathrm{b}}$

Roger D. Yusen, $\mathrm{MD}^{\mathrm{b}}$

Mary S. Pohl, RN ${ }^{\mathrm{a}}$

Stephen S. Lefrak, MD ${ }^{\mathrm{b}}$
Between January 1993 and February 1996, we performed 150 bilateral lung volume reduction procedures for patients with severe emphysema. Patients were selected on the basis of severe dyspnea, increased lung capacity, and a pattern of emphysema that included regions of severe destruction, hyperinflation, and poor perfusion. Twenty percent to $30 \%$ of the volume of each lung was excised with the use of a linear stapler and bovine pericardial strips attached to buttress the staple line. Patients were between 36 and 77 years old, with an average 1-second forced expiratory volume of $25 \%$ of predicted, total lung capacity of $142 \%$ of predicted, and residual volume of $\mathbf{2 8 3 \%}$ of predicted. Ninety-three percent of patients required supplemental oxygen, continuously or with exertion. All patients but one were extubated at the end of the procedure. The 90-day mortality was $4 \%$. Hospital stay progressively decreased with experience, and for the last 50 patients the median hospital stay was 7 days. Prolonged air leakage was the major complication. Results at 6 months show a $51 \%$ increase in the 1-second forced expiratory volume and a $28 \%$ reduction in the residual volume. The $\mathrm{PaO}_{2}$ increased by an average of $8 \mathrm{~mm} \mathrm{Hg}$, and $70 \%$ of the patients who had previously required continuous supplemental oxygen no longer had this requirement. The improvements in measured pulmonary function were paralleled by a significant reduction in dyspnea and an improvement in the quality of life. Reevaluation at 1 year and 2 years after operation showed the benefit to be well maintained. We conclude that lung volume reduction offers benefits not achievable by any means other than lung transplantation for highly selected patients with severe emphysema. (J Thorac Cardiovasc Surg 1996;112:1319-30)
$\mathrm{C}$ hronic obstructive pulmonary disease resulting from emphysema is a major cause of morbidity and mortality. ${ }^{1,2}$ As the disease progresses, physical activities become increasingly limited, and patients in advanced stages become dyspneic with minor

From the Divisions of Cardiothoracic Surgery ${ }^{\mathrm{a}}$ and Pulmonary and Critical Care Medicine, ${ }^{\mathrm{b}}$ Washington University School of Medicine, St. Louis, Mo.

Read at the Seventy-sixth Annual Meeting of The American Association for Thoracic Surgery, San Diego, Calif., April 28-May 1, 1996.

Received for publication May 6, 1996; revisions requested June 3, 1996; revisions received July 12, 1996; accepted for publication July 15, 1996.

Address for reprints: Joel D. Cooper, MD, Suite 3108, Queeny Tower, One Barnes Hospital Plaza, St. Louis, MO 63110.

Copyright $(\mathcal{1} 1996$ by Mosby-Year Book, Inc.

$0022-5223 / 96 \$ 5.00+0 \quad \mathbf{1 2 / 6 / 7 6 5 1 2}$ exertion or even at rest. Many patients require supplemental oxygen. The major therapeutic modalities consist of bronchodilator and antiinflammatory drugs, ${ }^{3,4}$ directed at decreasing airway resistance, and antibiotics to treat acute and chronic infection. Pulmonary rehabilitation techniques and exercise training have produced significant palliation. ${ }^{5}$ Supplemental oxygen therapy for the hypoxemic patient improves exercise performance and improves survival in patients with cor pulmonale. ${ }^{6,7}$ Despite all available therapies, the course of the disease is one of progressive limitation, increasing dyspnea, and significant increase in overall mortality. After the 1-second forced expiratory volume $\left(\mathrm{FEV}_{1}\right)$ falls below $30 \%$ of the predicted value, the overall survival at 3 years is only $60 \% .^{8,9}$

Emphysema interferes with respiratory function because of parenchymal destruction and changes in 
Table I. Eligibility criteria for evaluation for lung volume reduction surgery

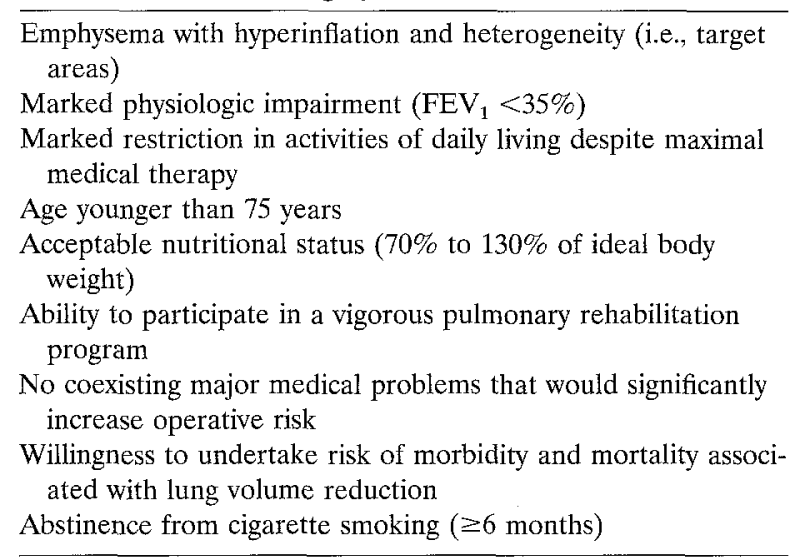

the mechanics of respiration. The progressive loss of lung elastic recoil leads to a decrease in the expiratory flow rate and to progressive overexpansion of the lung, with an increase in residual volume and total lung capacity. Because of the progressive increase in thoracic volume, chest wall and diaphragmatic function become impaired, increasing the work of breathing.

The lung volume reduction procedure is based on the proposals and the limited experience reported by Dr. Otto Brantigan more than 35 years ago. ${ }^{10} \mathrm{He}$ suggested that excision of some of the most destroyed portions of the lung could improve elastic recoil, reduce airflow limitation, and improve the mechanics of respiration. His limited experience with unilateral lung volume reduction, combined with the radical hilar stripping that was then in vogue, produced subjective improvements in a number of his patients. On the basis of Brantigan's proposals and our own observations of improved chest wall and diaphragmatic position after lung transplantation in patients with severe emphysema, we initiated a program for bilateral lung volume reduction in highly selected emphysema patients. This study was undertaken to evaluate the intermediate-term results of this procedure at our center.

\section{Methods}

Patient population. Between January 1993 and February 1996, 150 patients underwent bilateral lung volume reduction at the Washington University Medical Center. One hundred forty-nine procedures were carried out through a median sternotomy, and one procedure, in a patient who had previously undergone coronary bypass surgery, used bilateral muscle-sparing thoracotomies. One additional patient underwent median sternotomy for a
Table II. Reasons for declining patients for lung volume reduction surgery

\begin{tabular}{lr} 
Diffuse disease (no target areas) & $30 \%$ \\
Insufficient thoracic distention & $16 \%$ \\
Age or associated medical problems & $16 \%$ \\
$\mathrm{FEV}$, is too good & $8 \%$ \\
Pleural disease & $8 \%$ \\
Better suited for lung transplantation & $8 \%$ \\
$\mathrm{PaCO}_{2}>55$ (in association with other problems) & $7 \%$ \\
Marked kyphosis & $4 \%$ \\
Other & $3 \%$ \\
\hline
\end{tabular}

bilateral procedure, but extensive bilateral pleural adhesions were encountered and significant air leakage occurred after completion of volume reduction on the right side. Therefore the procedure was terminated without the contralateral reduction. This patient had a satisfactory result but is not included in this series.

Eligibility criteria include significant dyspnea and respiratory limitation despite maximum medical management, lung hyperinflation, and a favorable anatomic situation in which zones of relatively destroyed, hyperinflated lung coexist with other regions of lesser destruction. This pattern affords the opportunity of excising $20 \%$ to $30 \%$ of the volume of each lung, consisting of nonfunctional regions, to improve respiratory mechanics and distribution of the ventilation to the remaining lung. Several patients with diffuse disease and severe hyperinflation are included in this series.

The evaluation process, including physiologic and radiologic criteria, has been reviewed. ${ }^{11,12}$ In summary, thoracic distention is evaluated by inspiratory and expiratory chest radiographs, distribution of function by quantitative ventilation/perfusion lung scanning, and relative degree of parenchymal destruction in the various regions by computed tomography (CT) scanning of the chest. Eligibility criteria are summarized in Table I. None of these criteria was considered absolute, and exceptions were made for individual circumstances.

Approximately $80 \%$ of patients referred for this procedure were excluded from surgery, and the reasons for exclusion in more than 1000 evaluations are listed in Table II. Patients thought to be suitable candidates after initial review of records and on-site evaluation were enrolled in an exercise rehabilitation program for 6 weeks or longer. A final decision to proceed with the operation was made only after satisfactory completion of this program. The program included progressive exercise training and nutritional counseling. Nutritional supplements or diets were prescribed as necessary to adjust body weight to within $20 \%$ of the calculated ideal value. The preoperative phase of pulmonary rehabilitation began as soon as the patient was accepted for lung volume reduction. The goals included optimizing exercise endurance and pulmonary hygiene. All patients were placed in pulmonary rehabilitation programs close to their homes. The local program was provided exercise guidelines that included having the patients exercise 5 to 7 days each week, with the goal of completing 30 minutes of continuous exercise, preferably on the treadmill. 
Oxygen saturation of hemoglobin, as demonstrated by pulse oximetry, was maintained at $90 \%$ or greater with supplemental oxygen given as needed. The patient was provided as much oxygen as necessary to accomplish this task. On rare occasions oxygen concentrations approaching $100 \%$ delivered through a nonrebreather mask were used. The oxygen concentration was reduced immediately after the exercise. Treadmill speed was increased at subsequent exercise sessions, with the goal of attaining a heart rate in the target range $[(220-$ age $) \times(75 \%$ to $85 \%)$ ]. The guidelines also included optimizing pulmonary hygiene as necessary with the use of arm and leg weights to strengthen extremities and doing arm ergometry for 5 to 10 minutes to train the upper extremities, with resistance added if tolerated. The local pulmonary rehabilitation therapist was asked to send a facsimile of an exercise summary sheet to the lung volume reduction center every 2 weeks to determine the patient's progress and allow us to suggest changes to optimize the training program.

The physiologic profile of patients undergoing this procedure is shown in Table III. Pulmonary function studies were measured with a Medgraphics 1085 apparatus (Medical Graphics Corp, St. Paul, Minn.) before and after administration of aerosolized albuterol. The highest values obtained for forced vital capacity (FVC) and $\mathrm{FEV}_{1}$ were chosen for data analysis. Lung volumes were measured with a Medgraphic 1085 plethysmograph. Oxygen requirement at rest was defined as a requirement for supplemental $\mathrm{O}_{2}$ to maintain oxygen saturation at $88 \%$ or better while at rest (NELLCOR N10 pulse oximeter, Nellcor Inc., Pleasanton, Calif.). Oxygen requirement with exercise was defined as the maximum amount of supplemental oxygen required to maintain saturation at $90 \%$ or better during the 6-minute test. Dyspnea was quantified by the modified Medical Research Council (MRC) of Great Britain Dyspnea Scale ${ }^{13}$ and the Mahler Dyspnea Index. ${ }^{14}$ The modified MRC scale has five grades that describe the activity that provokes dyspnea; lower numbers correlate with less dyspnea. On the Mahler index, functional impairment is graded at baseline, but in contrast to the $\mathrm{MRC}$, higher numbers correspond to less dyspnea. A transition index was used to assess changes from the baseline value at a later date.

The typical patient experienced dyspnea at rest or with only mild exertion. Taking a shower, walking stairs, going shopping, and even getting dressed were associated for most patients with significant dyspnea that limited or prohibited the activity. Quality-of-life assessment was made by using two instruments, the Nottingham Health Profile ${ }^{15}$ and the Medical Outcomes Survey-Short Form 36. ${ }^{16}$

Statistical analysis. Pulmonary function data were analyzed with paired Student's $t$ test. Quality-of-life questionnaires were analyzed with repeated measures of analysis of variance and Tukey's pairwise comparisons to examine significant time effects.

Operative technique. A left-sided double-lumen tube was used to provide isolated ventilation to either lung. Before induction of anesthesia, a thoracic epidural catheter was placed under fluoroscopic guidance. This was used to reduce the amount of narcotic required intraoperatively and to provide optimal postoperative pain relief
Table III. Physiologic profile of 150 bilateral lung volume reduction patients

\begin{tabular}{ll}
\hline \multicolumn{1}{c}{ Characteristic } & \multicolumn{1}{c}{ Mean } \\
\hline Age (yr) & 61 (range 36 to $77 \mathrm{yr})$ \\
$\mathrm{FEV}_{1} \mathrm{~L} \mathrm{( \%} \mathrm{pred)}$ & $0.70(25 \%)$ \\
$\mathrm{FVC} \mathrm{L} \mathrm{( \%} \mathrm{pred)}$ & $2.5(70 \%)$ \\
$\mathrm{TLC} \mathrm{L} \mathrm{( \%} \mathrm{pred)}$ & $8.3(142 \%)$ \\
$\mathrm{RV} \mathrm{L} \mathrm{( \%} \mathrm{pred)}$ & $5.9(283 \%)$ \\
$\mathrm{PaO}_{2}(\mathrm{~mm} \mathrm{Hg})$ & 62 (range 36 to 109) \\
$\mathrm{PaCO}_{2}(\mathrm{~mm} \mathrm{Hg})$ & 42 (range 28 to 70$)$ \\
$\mathrm{O}_{2}$ required at rest (\% of patients) & $54 \%$ \\
$\mathrm{O}_{2}$ required with exercise (\% of pa- & $93 \%$ \\
$\quad$ tients) & $47 \%$ \\
Steroid dependent (\% of patients) & \\
\hline
\end{tabular}

FEV $V_{7}$, Forced expiratory volume in 1 second; pred, predicted; TLC, total lung capacity; $R V$, residual volume; $P a$, arterial partial pressure.

with a minimal need for narcotics or respiratory depressants. The technique was designed to permit extubation at the end of the procedure, and this goal was accomplished for all patients, except one patient who was successfully extubated the following morning. The anesthetic management used for these patients has been described elsewhere. ${ }^{17}$

The operative technique has evolved over the past 3 years. A linear stapler was used to excise the desired portions of the lung. In the first several patients, intraoperative air leaks were apparent at the proximal staple line when reexpansion of the lung caused tears in the visceral pleura at the staple puncture sites. This finding was associated with prolonged postoperative air leaks. The technique of reinforcing the staple line with strips of bovine pericardium (Peri-strips, Bio-Vascular, Inc., St. Paul, Minn.) attached to the surfaces of the stapler before its application virtually eliminated air leakage at the staple line. This technique has previously been reported. ${ }^{18}$ In the early part of this series, multiple independent wedge excisions were performed. However, the intervening pleura between such staple lines was often tense and subject to injury and rupture. To avoid this problem most resections are now performed with a continuous line of excision with successive application of the linear stapler. This operative technique has been reported elsewhere. ${ }^{19}$

In 15 patients $(10 \%)$, a complete anatomic lobectomy was used as part of the volume reduction procedure. Indications included known lung cancers in two and intraoperative findings of complete lobar destruction with a technically favorable situation for a lobectomy in the remaining patients.

The major focus of disease in most patients was in the upper lobes, in keeping with the known pattern of centrilobular emphysema in smokers. However, the region of most severe destruction in 18 patients was in the lower lobes, including 11 with known $\alpha_{1}$-antitrypsin deficiencies and 7 with normal enzyme levels.

A residual space at the apex of the chest is not uncommon after the volume reduction procedure. We have often created an apical pleural tent so that parietal and visceral pleura can be in apposition, even if the residual lung fails to expand sufficiently to completely fill 
Actuarial Survival

Volume Reduction Surgery $(N=150)$

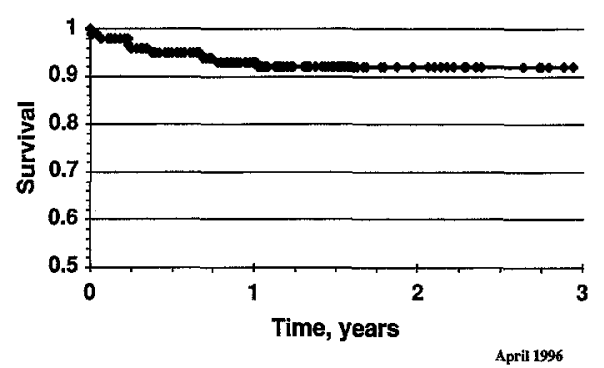

Fig. 1. Kaplan-Meier survival curve for 150 patients after lung volume reduction. Survival is $93 \%$ at 1 year and $92 \%$ at 2 years.

the chest. Although we have not evaluated the value of such a procedure in a randomized fashion, creation of a pleural tent is recommended when a sizable apical space remains at the end of the procedure. Two $28 \mathrm{~F}$ chest tubes are placed in each pleural space. Tubes are brought out through the upper abdomen in a subxiphoid position.

Our postoperative management was previously reviewed. ${ }^{19}$ The most significant change during the course of this series was the avoidance of suction on the chest tubes in the postoperative period. All tubes are attached to a water-seal drainage system. Suction is applied only if the postoperative chest $\mathrm{x}$-ray film reveals a pneumothorax greater than $30 \%$; the underlying lung appears restricted or compressed, as evidenced by increased density on the $\mathrm{x}$-ray film; or marked subcutaneous emphysema develops. Only $10(20 \%)$ of the last 50 patients required chest tube suction at any time in the postoperative period. If a modest air leak persisted beyond the first 4 to 5 postoperative days, the chest tube was connected to a Heimlich valve to facilitate patient mobility and discharge from the hospital.

The continuous epidural administration of bupivacaine, initiated intraoperatively, was continued in the recovery room, and a patient-controlled analgesia system (Baxter Healthcare Corp., Deerfield, Ill.) that delivered morphine sulfate $(80 \mathrm{mg} / 40 \mathrm{ml})$ was initiated. Several hours later, if further pain control was required, ketorolac was administered systemically, with an initial dose of $30 \mathrm{mg}$ followed by 15 to $30 \mathrm{mg}$ every 6 hours for 48 hours. The sympathectomy effect frequently associated with the epidural bupivicaine was counteracted as necessary with a continuous infusion of phenylephrine for 24 to 48 hours. Antibiotics, usually a cephalosporin, were administered intravenously for 5 days on a routine basis. Antibiotic coverage was broadened at the first suggestion of pulmonary infection. A team of chest physiotherapists supplemented the efforts of the experienced thoracic surgical nurses to provide vigorous chest physiotherapy and early ambulation. Patients began ambulation on a mobile treadmill brought to the bedside on the day after the operation. This permitted early ambulation of the patient without the

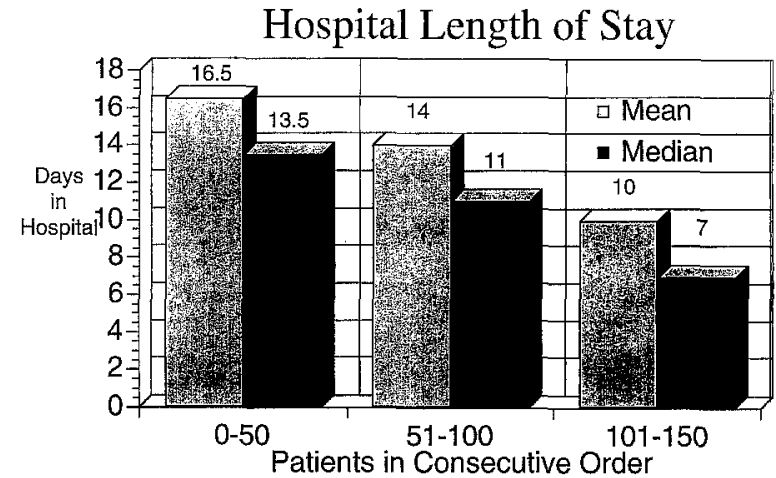

Fig. 2. Mean and median hospital stay for successive groups of 50 patients after bilateral lung volume reduction.

need to provide portable oxygen and monitoring equipment or to disconnect the patient from the many attachments. Despite an intensive program of chest physiotherapy, we found it useful to insert a minitracheostomy tube (Mini-Trach II, Portex, Keene, N.H.) for tracheal suction, because many of these patients had thick secretions and a feeble cough. In the last 50 patients in this series, minitracheostomy was used in $11(22 \%)$.

\section{Results}

Morbidity, mortality, and complications. Operative mortality was $4 \%$ (6 patients) and included all deaths from any cause occurring within 90 days of operation or at a later date if the patient was never discharged from the hospital or a chronic care facility. Late deaths were defined as any death occurring more than 90 days after the procedure for patients who had been discharged from hospital or a chronic care facility. Four such late deaths occurred in this series: one at 92 days and one at 270 days, both of pneumonia; one at 235 days of a stroke; and one at 13 months of respiratory failure. Mean follow-up time for survivors was 14 months and the median follow-up time 13 months. Actuarial survival, shown in Fig. 1, and was 93\% at 1 year and $92 \%$ at 2 years.

With experience and the changes in operative technique and postoperative management previously outlined, postoperative hospital stay progressively diminished, as illustrated in Fig. 2. All patients were discharged to their homes or to a hotel or self-care lodging facility if remaining in the St. Louis area for an additional week of observation and exercise training. For the group of 150 patients, the mean postoperative hospital stay was 13.5 days, with a median of 10 days. For the last 50 patients in this 


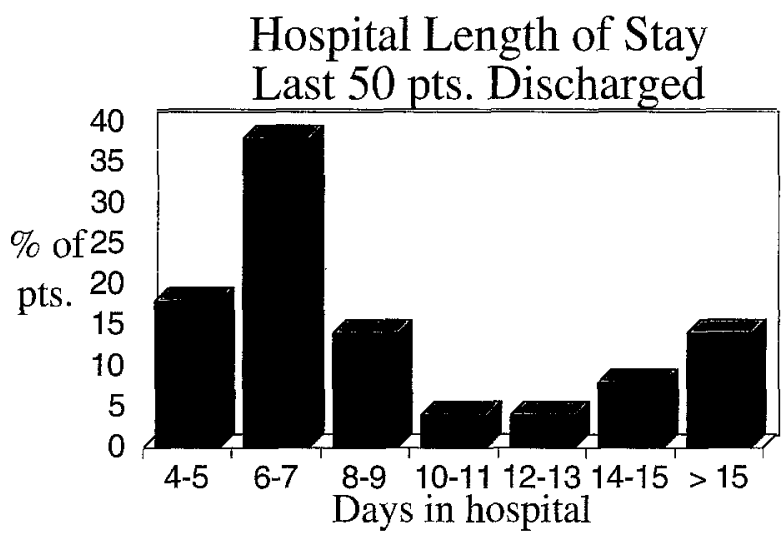

Fig. 3. Histogram of postoperative hospitalization for the last group of 50 patients after bilateral lung volume reduction.

series, the mean hospital stay was 10 days, with a median of 7 days (Fig. 3). Of these 50 patients, 54\% were discharged within 7 days and $67 \%$ within 9 days after the operation. Four patients with prolonged air leakage returned to their home cities with chest tubes and Heimlich valves in place. These tubes were removed at $18,35,37$, and 38 days postoperatively.

The significant hospital complications are listed in Table IV. Reexploration for air leakage occurred early in the series, when continuous and progressively increasing levels of chest suction were used to eliminate or reduce air spaces. No reexploration for air leakage or space problems has occurred in the last 75 patients. The duration of prolonged air leakage has diminished with avoidance of routine chest tube suction. In the last 50 patients, persistent air leakage ( $>7$ days) occurred in 18 patients $(36 \%)$.

Eleven patients required institution of mechanical ventilatory assistance at some time during their hospital stay. The duration of intubation ranged from 1 to 115 days. Five patients progressed to tracheostomy, and three of these ultimately died in the hospital.

Functional results. Median follow-up time was 415 days. Clinical follow-up information was available for all surviving patients, and postoperative measurement of pulmonary function at 3 or 6 months was available for all but 1 of the 127 patients who survived 6 months or longer after the operation.

After lung volume reduction, maximum improvement in measured lung function appeared to occur between 3 and 6 months preoperatively. Table V demonstrates the improvement seen in spirometry,
Table IV. Hospital complications for 150 patients

\begin{tabular}{lc}
\hline \multicolumn{1}{c}{ Complication } & Number of patients $(\%)$ \\
\hline Prolonged air leak ( $>$ 7 days) & $69(46)$ \\
Pneumonia & $17(11)$ \\
Mechanical ventilation & $11(7)$ \\
Reoperation & $9(6)$ \\
Air leak & 6 \\
Bleeding & 2 \\
Space problem & 1 \\
Tracheostomy & $5(3.3)$ \\
Myocardial infarction & $2(1.3)$ \\
Cardiac arrest & $2(1.3)$ \\
Cecal perforation & $2(1.3)$ \\
Bleeding into pleural tent & $2(1.3)$
\end{tabular}

lung volumes, and gas exchange 6 months after the procedure. The $\mathrm{FEV}_{1}$ increased by an average of $51 \%$, the residual volume declined by $28 \%$, and the mean $\mathrm{PO}_{2}$ increased by $8 \mathrm{~mm} \mathrm{Hg}$. Seventy percent of the patients who required continuous oxygen administration preoperatively were relieved of this necessity. Fifty-two percent of the patients who formerly required supplemental oxygen with maximum exertion required none at 6 months. This requirement was determined during the most vigorous pace of walking possible. Many patients who require oxygen under those circumstances do not require oxygen during routine, submaximal exertion. Overall functional improvement was measured with the 6-minute walk test. The results are shown in Table VI. Exercise capacity was significantly improved by the rehabilitation program, as has previously been demonstrated. ${ }^{20}$ After the procedure there was a further significant increase in overall performance. Oxygen requirements during exercise increased during the preoperative rehabilitation phase because of the higher achievable level of activity without concomitant improvement in pulmonary function. After surgery, oxygen requirements during exercise diminished significantly despite further significant increases in exercise capacity.

\section{Functional results}

Dyspnea. The level of dyspnea, as subjectively assessed by the patient, is depicted in Table VII. It was evaluated with the MRC scale and the dyspnea index of Mahler. Both showed a significant reduction of dyspnea at 6 months.

Quality of life, as assessed by the Medical Outcomes Survey-Short Form 36 and the Nottingham Health Profile, was evaluated when the patient was initially seen, after the preoperative rehabilitation program, and at intervals during the 
Table V. Results at 6 months for 101 patients

\begin{tabular}{|c|c|c|c|}
\hline Characteristic & Preoperative value & Postoperative value & Change \\
\hline $\mathrm{FEV}_{1} \mathrm{~L}(\%$ pred $)$ & $0.70(25 \%)$ & $1.06(38 \%)$ & $\uparrow 51 \% *$ \\
\hline FVC L (\% pred) & $2.5(69 \%)$ & $3.0(87 \%)$ & $\uparrow 20 \% *$ \\
\hline TLC L (\% pred) & $8.4(143 \%)$ & $7.2(125 \%)$ & $\downarrow 14 \% *$ \\
\hline RV L (\% pred) & $6.0(288 \%)$ & $4.3(205 \%)$ & $\downarrow 28 \% *$ \\
\hline $\mathrm{PaO}_{2}(\mathrm{~mm} \mathrm{Hg})$ & 62 & 70 & $\uparrow 8^{*}$ \\
\hline $\mathrm{PaCO}_{2}(\mathrm{~mm} \mathrm{Hg})$ & 43 & 39 & $\downarrow 4^{*}$ \\
\hline $\mathrm{O}_{2}$ required at rest (\% of patients) & $52 \%$ & $16 \% *$ & \\
\hline $\mathrm{O}_{2}$ required with exercise (\% of patients) & $92 \%$ & $44 \% *$ & \\
\hline Six-minute walk $(\mathrm{ft})$ & 1125 & $1311 *$ & \\
\hline Steroid dependent ( $\%$ of patients) & $49 \%$ & $18 \% *$ & \\
\hline
\end{tabular}

$F E V_{l}$, Forced expiratory volume in 1 second; pred, predicted; $F V C$, forced vital capacity; $T L C$, total lung capacity; $R V$, residual volume; $P a$, arterial partial pressure.

${ }^{*} p<0.001$.

Table VI. Six-minute walk

\begin{tabular}{lccrrr}
\hline & \multicolumn{2}{c}{ Preoperative } & & \multicolumn{2}{c}{ Postoperative } \\
\cline { 2 - 3 } \cline { 5 - 6 } & Before rehab & After rehab & & 3 mo & 6 mo \\
\hline Number of patients & 148 & 150 & & 81 & 94 \\
Distance & 856 & 1110 & & 1280 & 1316 \\
Percent of patients & $82 \%$ & $95 \%$ & & $47 \%$ & $43 \%$ \\
$\quad$ requiring $\mathrm{O}_{2}$ & & & & \\
\hline
\end{tabular}

Table VII. Subjective assessment of dyspnea by the Medical Research Council Scale and Mahler Index

\begin{tabular}{llll}
\hline & \multicolumn{2}{c}{ Preoperative } & Postoperative \\
\cline { 2 - 4 } Scale & Before rehab & After rehab & $(6$ mo $)$ \\
\hline Medical Research Council & 2.9 & 2.8 & 1.2 \\
Mahler Dyspnea Index & $0.92^{*}$ & $0.83 \dagger$ & $2.2 \uparrow$ \\
$\quad$ (functional impairment) & & & \\
*Baseline index. & & & \\
$\dagger$ Transitional index. & &
\end{tabular}

postoperative period. Only physical mobility, energy, and vitality showed improvement during the preoperative rehabilitation phase. Six months after the operation, there was further statistically significant improvement $(p<0.05)$. In these same parameters and in the other parameters listed in Table VIII.

One question contained in the Medical Outcomes Survey-Short Form 36 questionnaire asks how the patient views his or her overall health status compared with 1 year earlier. The responses for 108 patients 6 months after the procedure are shown in Table IX. Ninety-eight percent of patients felt better after surgery compared with 1 year earlier.

Intermediate term results. Of the 76 patients followed 1 or more years after the operation, 56 had pulmonary function measured at 6 months and at 1
Table VIII. Quality of life: items showing improvement at 6 months after lung volume reduction

Physical mobility*

Energy*

Emotional reaction

Job or work

Looking after home or home life

Sex life

Interests \& hobbies; enjoyment of holidays

Sleep

Vitality*

Physical functioning

General health

Social functioning

Mental health

"Changed to a significant degree $(p<0.05)$ during preoperative pulmonary rehabilitation, with further increase at 6 months postoperatively.

Table IX. Medical Outcome Survey-Short Form 36: response of 108 patients 6 months after lung volume reduction

\begin{tabular}{lc}
\hline \multicolumn{1}{c}{ Answer $^{*}$} & Patients responding (\%) \\
\hline Much better & 78 \\
Somewhat better & 20 \\
About the same & 1 \\
Somewhat worse & 1 \\
Much worse & 0
\end{tabular}

*Question: compared with 1 year ago, how would you rate your health in general now?

year. These data, shown in Table $\mathrm{X}$, indicated that the initial benefit was maintained at 1 year.

Our initial report ${ }^{21}$ presented our early experience with 20 patients. These 20 patients have been followed for a minimum of 24 months (range 25 to 39 months; mean 30 months). At last follow-up all patients still considered themselves to be signifi- 
Table X. Results of lung volume reduction for 56 patients

\begin{tabular}{lccc}
\hline \multicolumn{1}{c}{ Characteristic } & Preoperative value & Value at 6 months & Value at 1 year \\
\hline FEV 1 (\% pred) & $.69(24 \%)$ & $1.1(38 \%)$ & $1.0(36 \%)$ \\
FVC L (\% pred) & $2.4(67 \%)$ & $3.0(87 \%)$ & $3.0(85 \%)$ \\
TLC L (\% pred) & $8.1(139 \%)$ & $7.1(123 \%)$ & $7.3(125 \%)$ \\
$\mathrm{RV} \mathrm{L} \%$ pred) & $5.7(283 \%)$ & $4.1(201 \%)$ & $4.3(208 \%)$ \\
$\mathrm{PaO}_{2}$ (mm Hg) & 63 & 72 & 71 \\
$\mathrm{PaCO}_{2}$ (mm Hg) & 43 & 38 & 37 \\
$\mathrm{O}_{2}$ required at rest (\% of patients) & $58 \%$ & $9 \%$ & $16 \%$ \\
$\mathrm{O}_{2}$ required with exercise (\% of patients) & $92 \%$ & $38 \%$ & $51 \%$ \\
Six-minute walk (ft) & 1150 & 1362 & 1357 \\
Steroid dependent (\% of patients) & $53 \%$ & $17 \%$ & $19 \%$ \\
\hline
\end{tabular}

$F E V_{1}$, Forced expiratory volume in 1 second; pred, predicted; $F V C$, forced vital capacity; $T L C$, total lung capacity; $R V$, residual volume; $P a$, arterial partial pressure.

Table XI. Results of the initial 20 patients

\begin{tabular}{|c|c|c|c|}
\hline Characteristic & Preoperative value & Value at 1 year & Most recent value \\
\hline $\mathrm{FEV}_{1} \mathrm{~L}(\%$ pred $)$ & $.83(27 \%)$ & $1.33(45 \%)$ & $1.25(42 \%)$ \\
\hline FVC L (\% pred) & $2.6(69 \%)$ & $3.2(88 \%)$ & $3.3(87 \%)$ \\
\hline TLC L (\% pred) & $8.4(141 \%)$ & $7.7(131 \%)$ & $7.6(127 \%)$ \\
\hline RV L (\% pred) & $5.9(291 \%)$ & $4.4(212 \%)$ & $4.4(212 \%)$ \\
\hline $\mathrm{PaO}_{2}(\mathrm{~mm} \mathrm{Hg})$ & 64 & 73 & 73 \\
\hline $\mathrm{PaCO}_{2}(\mathrm{~mm} \mathrm{Hg})$ & 44 & 37 & 39 \\
\hline $\mathrm{O}_{2}$ required at rest ( $\%$ of patients) & $26 \%$ & $0 \%$ & $0 \%$ \\
\hline $\mathrm{O}_{2}$ required with exercise ( $\%$ of patients) & $84 \%$ & $5 \%$ & $32 \%$ \\
\hline Six-minute walk $(\mathrm{ft})$ & 1205 & 1475 & 1481 \\
\hline Steroid dependent ( $\%$ of patients) & $42 \%$ & $6 \%$ & $11 \%$ \\
\hline
\end{tabular}

$F E V_{i}$, Forced expiratory volume in 1 second; pred, predicted; $F V C$, forced vital capacity; $T L C$, total lung capacity; $R V$, residual volume; $P a$, arterial partial pressure.

*Patients were followed for at least 24 months.

cantly improved over their preoperative status. The most recent follow-up data on these patients are shown in Table XI and confirm persistent objective benefit at 2 years.

\section{Discussion}

Our original report of bilateral lung volume reduction surgery in 20 patients showed encouraging early results. ${ }^{21}$ Subsequent experience has confirmed the benefit achieved with this procedure in selected patients and has indicated that subjective and objective improvement persist for at least 2 years. It is assumed that the progressive deterioration in lung function known to occur over time with emphysema will continue, but many patients continue to exhibit subjective and objective benefit, and five of the initial patients who have been followed 3 or more years show well-sustained benefit.

We consider the preoperative rehabilitation program to be essential for three reasons. First, a decision to operate should only be made after optimum medical management has proved insufficient. Second, the improved stamina and nutritional status facilitates postoperative recovery. It has not been uncommon for patients who are initially totally wheelchair dependent to progress to the point of 30 minutes of continuous exercise on a treadmill at 1 mile per hour for 30 minutes at the conclusion of the rehabilitation phase. Third, optimized preoperative medical management provides an appropriate baseline for evaluation of the benefits produced by the operation.

We use the median sternotomy because this approach provides excellent bilateral exposure, permits a bilateral approach through single incision, and allows continuous surveillance of both pleural cavities throughout the procedure. In tandem with our analgesic protocol, we believe median sternotomy is associated with minimum morbidity. It is the ultimate muscle-sparing incision, because none of the chest wall muscles is incised, retracted, or penetrated. No painful intercostal nerve injury can result from the operative approach or from chest tube placement sites located in the subxyphoid region.

Miller and associates have reported an experience 
Table XII. Results after unilateral lung volume reduction for 17 patients

\begin{tabular}{|c|c|c|c|c|}
\hline Characteristic & Preoperative value & Value at 3 to 6 months & Change & $p$ Value \\
\hline $\mathrm{FEV}_{1} \mathrm{~L}(\%$ pred $)$ & $.81 \%(28 \%)$ & $1.08(39 \%)$ & $\uparrow 33 \%$ & $<.01$ \\
\hline FVC L ( $\%$ pred) & $2.7(75 \%)$ & $3.1(88 \%)$ & $\uparrow 15 \%$ & $<.05$ \\
\hline TLC L (\% pred) & $8.7(139 \%)$ & $7.4(125 \%)$ & $\downarrow 15 \%$ & $<.01$ \\
\hline $\mathrm{RV} L$ (\% pred) & $6.0(266 \%)$ & $4.4(193 \%)$ & $\downarrow 27 \%$ & $<.001$ \\
\hline $\mathrm{PaO}_{2}(\mathrm{~mm} \mathrm{Hg})$ & 63 & 71 & $\uparrow 8$ & $<.05$ \\
\hline $\mathrm{PaCO}_{2}(\mathrm{~mm} \mathrm{Hg})$ & 41 & 39 & & NS \\
\hline $\mathrm{O}_{2}$ required at rest (\% of patients) & $53 \%$ & $24 \%$ & & \\
\hline $\mathrm{O}_{2}$ required with exercise (\% of patients) & $94 \%$ & $71 \%$ & & \\
\hline Six-minute walk $(\mathrm{ft})$ & 1038 & 1134 & & $<.05$ \\
\hline Steroid dependent ( $\%$ of patients) & $50 \%$ & $36 \%$ & & \\
\hline
\end{tabular}

$F E V_{J}$, Forced expiratory volume in 1 second; pred, predicted; $F V C$, forced vital capacity; $T L C$, total lung capacity; $R V$, residual volume; $P a$, arterial partial pressure.

with bilateral volume reduction similar to our own. ${ }^{22}$ Several other teams have reported their experience with volume reduction conducted by a unilateral thoracoscopic approach. The results confirm the benefits of the volume reduction concept, although objective improvement of a lesser magnitude has generally been accomplished with this unilateral approach. ${ }^{23-25}$ As a result, a bilateral thoracoscopic approach has been proposed at some centers. Our own experience includes 24 cases of unilateral volume reduction, all undertaken because of contraindications to a bilateral procedure or because of a marked discrepancy in the degree of emphysematous changes in the two lungs. A muscle-sparing thoracotomy approach was used in these 24 patients. None of these patients has died, and significant benefit has been achieved, although of lesser magnitude than that achieved with the bilateral procedure (Table XII).

We have specifically excluded from this report patients who underwent excision of giant bullae. During the period in which we performed the 150 bilateral and 24 unilateral lung volume reduction procedures, only 9 patients have undergone bilateral giant bullectomy as indicated by the standard selection criteria, which are giant space-occupying bullae in the presence of "relatively normal underlying compressed lung," as reported by Connelly and coworkers $^{26}$ in 1989. Despite the heightened interest in operations for emphysema, the relatively small number of patients referred for this condition emphasizes the relative rarity of this entity.

Two of the major issues that remain unresolved for the lung volume reduction procedure are how to select appropriate candidates and how to assess and interpret the results. We continue to be quite conservative in our selection process, requiring significant functional limitation, marked thoracic disten- tion, and a favorable anatomic situation that permits excision of areas of marked destruction while retaining regions with lesser disease. With some exceptions, we have excluded the much greater proportion of patients whose emphysema is rather uniformly distributed. Although several such patients with satisfactory results are included in this series, it remains our assumption that the magnitude of improvement and the duration of benefit are related to the amount of lung with minimal to moderate disease that remains at the end of the procedure. To prove whether this assumption is correct would require further experience with liberalized selection criteria.

Objective evaluation of the degree and pattern of lung destruction remains imprecise. Despite considerable experience, our selection process admittedly contains a significant subjective component. Techniques are available to objectively quantify the degree and distribution of lung damage, such as highresolution CT scan or quantitative CT in which a computerized program breaks down each lung slice into small pixels and analyzes each pixel for lung density. Such an analysis is capable of expressing the percentage of lung in each region that has severe, moderate, or minimal destruction. However, this technique is of more theoretical than practical value.

In the current series, the most severely diseased lung usually was in the upper lobes, consistent with the known distribution of centrilobular emphysema in smokers. However, the most severe areas of destruction were located in the lower lobes of 18 patients. Eleven had $\alpha_{1}$-antitrypsin deficiency, and seven did not. In these 18 patients the mean improvement in $\mathrm{FEV}_{1}$ has been $27 \%$, the reduction in residual volume was $28 \%$, and the increase in $\mathrm{PaO}_{2}$ was $5 \mathrm{~mm} \mathrm{Hg}$. These values are significantly less 
than for the overall series, but nonetheless most of these patients have experienced significant functional improvement.

The physiologic alterations responsible for the subjective and functional improvement observed after lung volume reduction remain unclear. There is often a lack of correlation in the degree of objective improvement achieved with each of the parameters. For any individual patient a minor increase in the $F E V_{1}$ may be associated with a marked reduction in residual volume or with a very significant increase in the $\mathrm{PaO}_{2}$. The overall benefit, as perceived by the patient, occasionally correlates poorly with the changes in physiologic measurements. However, the significant improvement seen in virtually all objective parameters measured in the group as a whole confirms that the benefits perceived by the patients are related to alterations produced by the procedure and not to a "sham effect," such as has been associated with numerous procedures performed earlier in this century and for which no demonstrable objective improvement could be documented. It is probable that the measurements we have made do not fully reflect the physiologic alterations brought about by the volume reduction procedure. More sophisticated studies, including measurement of the work of breathing and lung elastic recoil, have been made by others and have shown significant improvement after the operation. $^{22,27,28}$

It has been suggested that the benefits achieved with the volume reduction procedure might accrue with a longer period of medical management and exercise rehabilitation and without an operative procedure. This is not the case. Each patient in this series served as his or her own control after receiving maximum medical therapy as outlined in the American Thoracic Society Guidelines for the management of chronic obstructive pulmonary disease. ${ }^{29}$ Other publications have documented that, notwithstanding the important benefits of a rehabilitation program in terms of increased mobility, stamina, and somewhat lessened dyspnea, no improvement in measurable lung function or gas exchange occurred as a result of such a program. ${ }^{9,30,31}$

A second question has been raised regarding the benefits of this procedure and the anticipated duration of improvement achieved. Only complete follow-up of these patients will provide an answer. We hope with this procedure to "turn back the clock" by a number of years, while recognizing that the natural progression of the underlying process will continue. Whether this progression will be at the rate usually anticipated, slower because of improved lifestyle and medical management, or accelerated because of the increased distending force on the residual diseased lung remains to be seen.

We have not yet undertaken a formal study of the ongoing medical costs associated with the management of these patients, but our patients report that visits to physicians and to emergency wards and the frequency of hospitalization have all been markedly diminished after the operation. Prolongation of life expectancy, although not the goal of lung volume reduction, appears to be another significant potential benefit. Given the known correlation between $\mathrm{FEV}_{1}$ and life expectancy, ${ }^{8,9}$ the mean improvement in $\mathrm{FEV}_{1}$ demonstrated in this series may have a significant impact on the patients' longevity. The actuarial survival of $92 \%$ at 24 months compares favorably with the anticipated death rate for patients with this degree of chronic obstructive pulmonary disease. However, this discrepancy may be related to a selection bias. Only a randomized, controlled study in which one half of the eligible patients are denied the volume reduction procedure for a number of years would address this issue from a rigorous scientific standpoint. However, given the results achieved, the absence of any alternative therapy for these patients, and the fact that improved quality of life, not longevity, is the goal of this procedure, such a randomized trial poses serious ethical and moral issues. One method for evaluating the impact of lung volume reduction on life expectancy would be to compare results achieved after lung volume reduction with the results for a cohort of patients who have been equivalently assessed for the procedure and found to be suitable in all respects except for the presence of a homogeneous pattern of destruction throughout the lungs. This would render them unsuitable for the volume reduction procedure by our current criteria. This cohort of patients would then be similar in all other respects, including severity of disease and absence of surgical contraindications, to the operative group and could serve as the best "natural history" control group.

The relationship between lung volume reduction and lung transplantation for severe emphysema remains an important issue. Approximately two thirds of the patients in this series would not have qualified for lung transplantation because of age, coexisting medical problems, or insufficient severity of disease to justify transplantation. Similarly, approximately two thirds of patients with obstructive 
lung disease evaluated for lung transplantation at our center are considered unsuitable for lung volume reduction, usually because of severe diffuse disease, associated small airways disease, or chronic infection. For the remaining patients, lung transplantation and lung volume reduction surgery can both be considered. Under these circumstances, the patients are informed of the risks and benefits of each procedure, and virtually all have chosen to undergo lung volume reduction, with the understanding that this does not, in our opinion, preclude the chances for successful lung transplantation should it subsequently be deemed appropriate.

At the time of this operation, 48 patients were considered suitable candidates for lung transplantation on the basis of age, severity of lung function, and absence of apparent contraindications. None of these patients has received a lung transplant. Four of the patients in this series are actively listed for a lung transplantation, and eight others, who were listed for a transplant before their operations, are not currently considered active candidates because of the improvement in lung function after the reduction procedure.

The lung volume reduction procedure has, temporarily at least, shifted a number of potential lung transplant recipients to an alternative procedure and has had the effect of increasing the number of available donors for patients suffering from other conditions, such as cystic fibrosis and pulmonary hypertension, for whom no alternative to transplantation exists.

The risks of lung volume reduction for disabling emphysema should not be underestimated. Selection, preparation, and postoperative care of these patients requires the coordinated activity of thoracic surgeons, anesthesiologists, pulmonologists, nurses experienced in the management of thoracic operative patients, respiratory therapists, and others. In the absence of such a team approach, the morbidity and mortality associated with this procedure may well exceed that which we have reported.

The lung volume reduction operation is logical, physiologically sound, and of proven benefit for a selected group of patients with no alternative therapy except lung transplantation, for which only a minority qualify. Further refinements in selection, operative technique, and postoperative management are anticipated. Accurate collection and reporting of data from different centers will permit a comparison of different selection criteria, various operative approaches and techniques, and refinements in postoperative management. Current re- sults confirm an important albeit palliative role for the lung volume reduction procedure.

We acknowledge the essential contributions made to the success of the lung volume reduction program by cardiothoracic anesthesia staff; the nursing service of the Division of Cardiothoracic Surgery; the chest physiotherapists and respiratory therapists, Dottie Biggar, RN, and the staff of the pulmonary rehabilitation team; Veronica Richardson Higgins, RN, the nurse coordinator; and many other members of the clinical team who provided experienced and tireless support for the complicated care for this group of patients. We also acknowledge Ms. Kathy Stroud for her expert secretarial support.

\section{REFERENCES}

1. Feinleib M, Rosenberg H, Collins J, et al. Trends in COPD morbidity and mortality in the United States. Am Rev Respir Dis 1989;140:S9-18.

2. Higgins M, Keller J. Trends in COPD morbidity and mortality in Tecumseh, Michigan. Am Rev Respir Dis 1989;140: S42-8.

3. Albert R, Martin T, Lewis S. Controlled clinical trial of methylprednisolone in patients with chronic bronchitis and acute respiratory insufficiency. Ann Intern Med 1980;92: 753-8.

4. Mendella L, Manfreda J, Warren C, et al. Steroid response in stable chronic obstructive pulmonary disease. Ann Intern Med 1982;96:17-21.

5. Petty T, Finigan M, Brink G, et al. A comprehensive care program for chronic airway obstruction: methods and preliminary evaluation of symptomatic and functional improvement. Ann Intern Med 1969;70:1109-20.

6. Nocturnal Oxygen Therapy Trial Group. Continuous or nocturnal oxygen therapy in hypoxemic chronic obstructive lung disease: a clinical trial. Ann Intern Med 1980;93:391-8.

7. Intermittent Positive Pressure Breathing Trial Group. Intermittent positive pressure breathing therapy of chronic obstructive pulmonary disease. Ann Intern Med 1983;99:61220.

8. Anthonisen NR. Prognosis in chronic obstructive pulmonary disease: results from multicenter clinical trials. Am Rev Respir Dis 1989;140:S95-9.

9. Ries AL, Kaplan RM, Limberg TM, Prewitt L. Effects of pulmonary rehabilitation on physiologic and psychosocial outcomes in patients with chronic obstructive pulmonary disease. Ann Intern Med 1995;12:823-32.

10. Brantigan OC, Mueller E, Kress MB. A surgical approach to pulmonary emphysema. Am Rev Respir Dis 1959;80:194-206.

11. Yusen RD, Lefrak SS, and The Washington University Emphysema Surgery Group. Evaluation of patients with emphysema for lung volume reduction surgery. Semin Thorac Cardiovasc Surg 1996;8:83-93.

12. Slone RM, Gierada DS. Radiology of pulmonary emphysema and lung volume reduction surgery. Semin Thorac Cardiovasc Surg 1996;8:61-82.

13. American Thoracic Society. Surveillance for respiratory hazards in the occupational setting. Am Rev Respir Dis 1982; 126:952-6.

14. Mahler D, Weinberg D, Wells C, et al. The measurement of 
Volume 112, Number 5

dyspnea: contents, interobserver agreement, and physiologic corrections of two new clinical indexes. Chest 1984;85:751-8.

15. Hunt S, McKenna S, McEvew J, et al. The Nottingham Health Profile: subjective health status and medical consultations. Soc Sci Med 1981;15A:221-9.

16. Ware J Jr, Sherbourne C. The MOS 36-item short-form health survey (SF-36): I. Conceptual framework and item selection. Med Care 1992;30:473-81.

17. Triantafillou AN. Anesthetic management for bilateral volume reduction surgery. Semin Thorac Cardiovase Surg 1996; 8:94-8.

18. Cooper JD. Technique to reduce air leaks after resection of emphysematous lung. Ann Thorac Surg 1994;57:1038-9.

19. Cooper JD, Patterson GA. Lung volume reduction surgery for severe emphysema. Semin Thorac Cardiovase Surg 1996; 8:52-60.

20. Holle RHO, Williams DV, Vandree JC, et al. Increased muscle efficiency and sustained benefits in an outpatient community hospital-based pulmonary rehabilitation program. Chest 1988;94:1161-8.

21. Cooper JD, Trulock EP, Triantafillou AN, et al. Bilateral pneumectomy (volume reduction) for chronic obstructive pulmonary disease. J Thorac Cardiovasc Surg 1995;109:106-19.

22. Miller DL, Dowling RD, McConnel JW, Skolnick JL. Effects of lung volume reduction surgery on lung and chest wall mechanics. Ann Thorac Surg. In press.

23. Keenan RJ, Landreneau RJ, Sciurba F, et al. Unilateral thoracoscopic surgical approach for diffuse emphysema. J Thorac Cardiovasc Surg 1996;111:308-16.

24. McKenna RJ, Brenner MA, Gelb AF, et al. A randomized prospective trial of stapled lung reduction versus laser bullectomy for diffuse emphysema. J Thorac Cardiovasc Surg 1996;111:317-22.

25. Naunheim KS, Keller CA, Krucylak PE, et al. Unilateral video-assisted thoracic surgical lung reduction. Ann Thorac Surg 1996;61:1092-8.

26. Connolly JE, Wilson A. Current status of surgery for bullous emphysema. J Thorac Cardiovasc Surg 1989;97:351-61.

27. Gelb AF, Zamel N, McKenna RJ, Brenner M. Mechanism for short term improvement in lung function following emphysema resection. Am J Respir Crit Care Med 1996;154: 945-51.

28. Sciurba FC, Rogers RM, Keenan RJ, et al. Improvement in pulmonary function and elastic recoil after lung reduction surgery for diffuse emphysema. N Engl J Med 1996;334: 1095-9.

29. American Thoracic Society. ATS statement for diagnosis and care of patients with chronic obstructive pulmonary disease. Am Rev Respir Crit Care Med 1995;152 Suppl 5:S78-121.

30. Casaburi R, Patessioa A, Iloi F, et al. Reductions in exercise lactic acidosis and ventilation as a result of exercise training in patients with obstructive lung disease. Am Rev Respir Dis 1991;143:9-18.

31. Biggar D, Malen J, Trulock E, Cooper J. Pulmonary rehabilitation before and after lung transplantation. In: Casaburi R, Petty TL, editors. Principles and practice of pulmonary rehabilitation. Philadelphia: WB Saunders, 1993:459-67.

\section{Discussion}

Dr. John R. Benfield (Sacramento, Calif.). Out of respect for the Program Committee's desire not to have slides shown, I selected three slides that I am not going to show you. The first shows Joel Cooper at the Toronto General Hospital with his first successful lung transplantation patient and our Past President, Griff Pearson; the second is a letter I received from one of our patients. The patient says that he is a 73-year-old farmer who is back mowing hay and playing with his grandchildren after lung reduction. The third is a letter from that same patient to our Senator, Diane Feinstein, telling her that he cannot understand why the Health Care Financing Agency (HCFA) is not paying for lung reduction. I admire Joel Cooper's boundless energy and enthusiasm for the treatment of end-stage lung disease.

The manuscript contains three points I would like to address. The first is that long-term outcome after lung volume reduction remains unknown. To this I say that the absence of such data is being ameliorated daily as shortterm and intermediate-length outcome data accumulate. The second point is that there are no data about medical costs of emphysema with the lung volume reduction procedure compared with nonoperative management, including pulmonary rehabilitation. To this I respond that we have eclipsed the past, when any new, beneficial health care measure was affordable. The need to contain cost requires that the value of lung volume reduction be measured by outcome divided by cost. We must determine whether the initial high cost of lung volume reduction is balanced by lesser long-term costs for medication and medical care for patients who are treated nonoperatively. The third point is that only about $20 \%$ of patients referred were accepted for operation. This underscores the need to undertake lung volume reduction as a team approach to avoid significantly higher morbidity and mortality than reported by the Washington University group.

I close with a comment about the current lack of Medicare funding for lung volume reduction and with a question. The organizations that are the national forums for science and continuing education in thoracic surgery and in pulmonary medicine have advised HCFA that they do not consider lung volume reduction experimental. Hospital costs and professional fees therefore should be paid by Medicare when the procedure is offered to properly selected patients in centers that have track records successfully conducted, credible clinical research. There is no doubt that further research on the treatment of end-stage lung disease needs to be done and that lung volume reduction is a prime example for HCFA and the National Institutes of Health (NIH) to come together. HCFA should fund the patient care aspects of the treatment, and the NIH should support the needed research costs. Organized thoracic surgery spearheaded by the Society of Thoracic Surgeons and supported by the American Association for Thoracic Surgery and respiratory medicine have stepped forward and offered help to HCFA and the NIH in this regard. I hope that these agencies will accept this offer on behalf of patients whose dyspnea deserves palliation. I believe that a randomized study in the early 1970 s to compare aortocoronary bypass to the then best available nonoperative treatment would have resulted in far quicker acceptance of the operation than occurred. We are at a similar stage with lung volume reduction, because it remains possible and perhaps likely 
that the benefits of lung volume reduction will prove to be transient.

Dr. Cooper, would it not be best to proceed with a randomized, prospective evaluation of lung volume reduction surgery compared with the best available pulmonary rehabilitation?

Dr. Cooper. Thank you, Dr. Benfield. You raised the issue of values. The scientific issues, such as how does this operation work, and how long will it last, all require careful scrutiny and the usual process by which, over the years, we decide which therapies are or are not of value. I believe these questions can be answered without randomization. The issues of value, such as how much is it worth to feel better and how long does the patient have to feel better to make it cost effective, probably cannot be answered by a randomized trial.

A randomized trial may not be the best approach for evaluating the medical benefits of the procedure. Lung volume reduction produces changes in $\mathrm{FEV}_{1}$, residual volume, and other objective measurements which cannot be achieved by any current nonsurgical treatment. Therein is the dilemma of denying it to patients for whom it is the only possible choice. I completely subscribe to careful scrutiny and evaluation, and I have no objection to any center conducting a compassionate and randomized trial as long as scientific evaluation is not used as a disguise for rationing or access limitation to a procedure for which there is no alternative.

Dr. Cecil C. Vaughn (Phoenix, Ariz.). I wish to comment on the technique of minimizing air leakage after the lung volume reduction procedure. I heartily endorse the need for staple line reinforcement and staple wedge resections of emphysematous lung. My colleagues and I use quadrangular sleeves of expanded polytetrafluoroethylene (PTFE)* that fit on the anvil and cartridge of the stapler. These sleeves have tear seams and grasping flaps at each end to facilitate removal of the excess PTFE after firing the stapler. These sleeves have been used in 21 open and thoracoscopic procedures at our center in Phoenix and at the University of Vienna, and they represent another technique for the reduction of air leak.

Dr. Cooper, what has been your experience with bovine pericardium, and how long did bovine pericardium last after its application?

Dr. Cooper. When we initially reported the use of bovine pericardium, we summarized many of the different patching techniques and buttressing techniques that have been documented by others over the years, and I suppose it is a matter of personal preference. I have had the opportunity to reexplore two patients a month or more after the initial procedıres, and the bovine pericardium seemed totally inert and well incorporated. I can only judge it on the basis of previous studies done during the last 10 or 15 years in whic: many persons have studied the material as a bioprosthesis and as a tissue support. I agree with you that some form of buttressing the staple line in emphysematous lungs produces benefit, but I am concerned about the use of nonabsorbable foreign material in the potential presence of a prolonged air leak or pleural infection.

* Seamguard Material, W. L. Gore \& Associates, Inc., Newark, Del. 